\title{
Successful treatment of an iatrogenic biliary stricture using holmium laser "recanalization" and multistenting technique
}

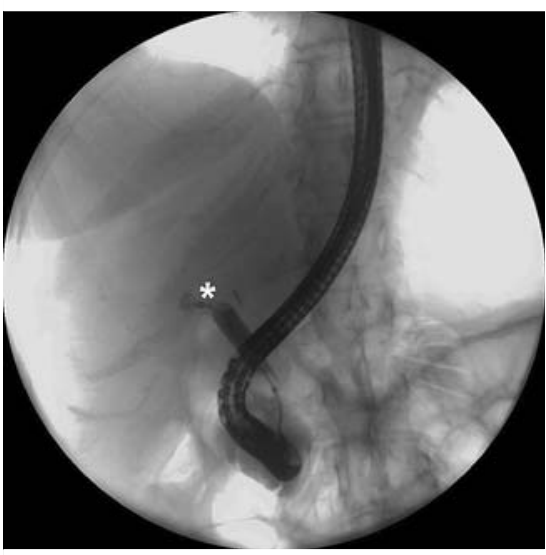

- Fig. 1 Cholangiography during endoscopic retrograde cholangiopancreatography showed a tight stricture of the proximal common bile duct (CBD; asterisk) near the projection of the surgical clips, with no passage of contrast medium into the intrahepatic bile ducts.

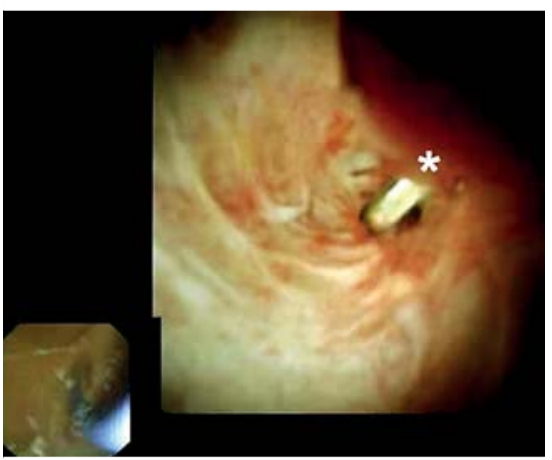

- Fig. 2 Cholangioscopy using SpyGlass DS revealed a complete stricture of the proximal CBD with the tip of a metallic clip (asterisk) clearly visible inside the CBD.

A 55-year-old woman admitted for jaundice 2 months after cholecystectomy underwent magnetic resonance imaging, which showed a common bile duct (CBD) stricture. Cholangiography during endoscopic retrograde cholangiopancreatography ( Fig. 1 ) confirmed a tight stricture of the proximal CBD and lack of visualization of the intrahepatic bile ducts (IHBD). At cholangioscopy using

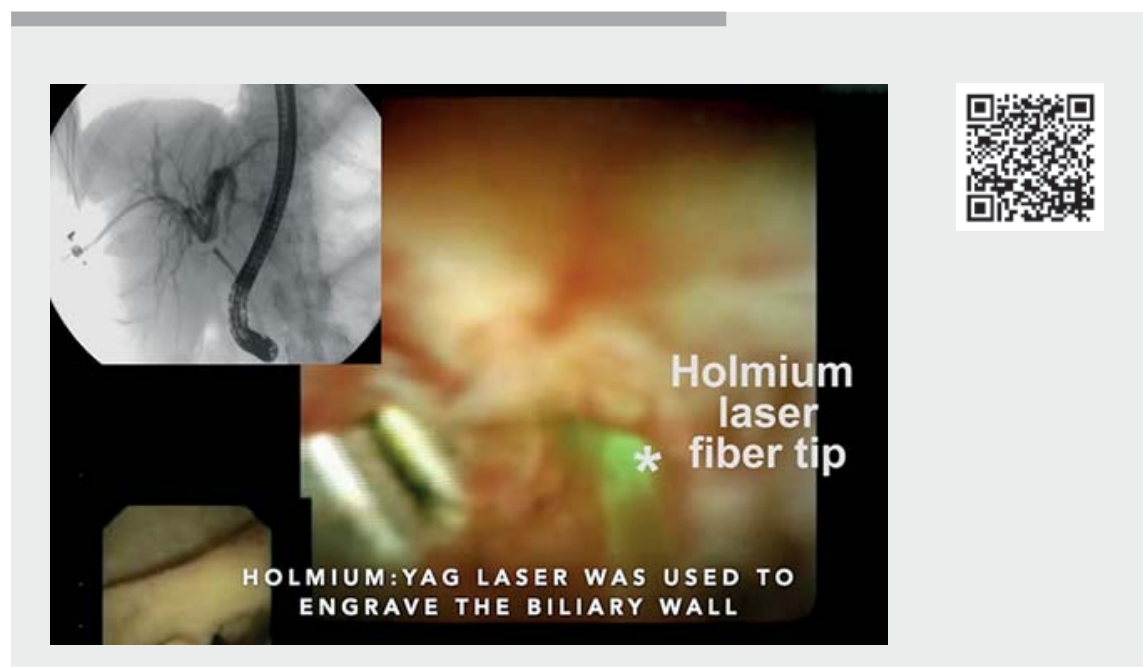

Video 1 The holmium:YAG laser fiber was oriented toward the biliary wall by radiologically following with SpyGlass the presumed anatomic direction of the CBD until "recanalization" of the stricture was achieved.

SpyGlass DS (Boston Scientific), a complete stricture of the proximal CBD was observed with a metallic clip visible inside the CBD ( $\triangleright$ Fig. 2 ). The on-call interventional radiologist performed a percutaneous transhepatic biliary drainage (PTBD) procedure in order to try a rendezvous approach. Percutaneous contrast medium injection confirmed dilated IHBD with complete absence of the proximal CBD lumen for a length of at least $5 \mathrm{~mm}$. Multiple attempts to pass a guidewire through the stricture, both percutaneously and endoscopically, failed.

We decide to use holmium:YAG laser to cut through the biliary wall ( $\vee$ Video 1 ) under cholangioscopic view by radiologically following the axis of SpyGlass and the presumed anatomic direction of the CBD until we were able to pass a guidewire into the IHBD. Cholangiography confirmed the passage of contrast medium through the "recanalized" CBD stricture (> Fig.3). After hydropneumatic balloon dilation of the stricture up to $8 \mathrm{~mm}$, the choledochoscope was able to pass

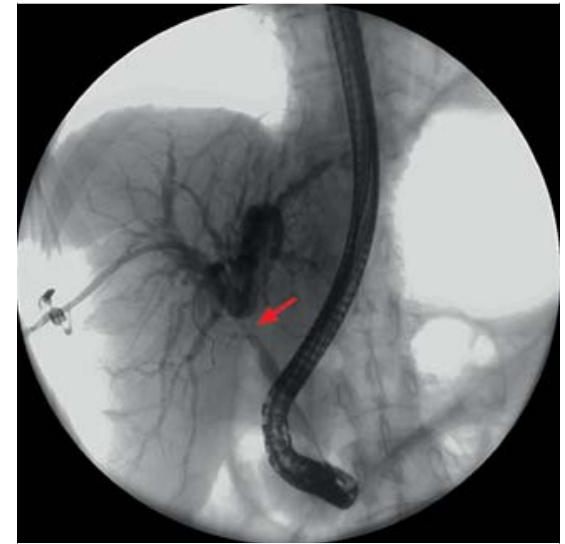

- Fig. 3 After laser treatment, cholangiography confirmed "recanalization" of the CBD stricture, with passage of contrast medium (arrow) through the stricture into the distal CBD, without spreading outside the CBD.

through the stricture ( $\mathbf{F i g}$.4) over the guidewire into the IHBD, directly visualizing the PTBD, which was finally removed. Lastly, four plastic biliary stents were placed bilaterally across the stricture. After an intermediate exchange and addi- 


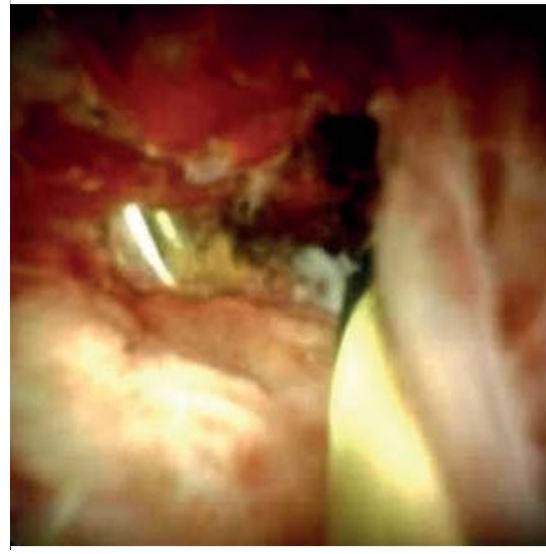

Dig. 4 Choledochoscopic view of the stricture after laser and dilation treatments, with visible clip and guidewire passed through the "recanalized" lumen of the CBD.

tion of two more stents, cholangiography 6 months later showed complete resolution of the stricture ( $>$ Fig. 5).

Endoscopic therapy is considered the first-line treatment for benign biliary stricture following cholecystectomy [13]. A multidisciplinary approach in collaboration with an interventional radiologist was useful [4] in managing this case of complete iatrogenic proximal CBD stricture, enabling the operator to be guided during the application of the holmium laser technique. In this way, "recanalization" of the CBD lumen was achieved, thus permitting the usual multistenting treatment to be carried out.

\section{Endoscopy_UCTN_Code_TTT_1AR_2AG}

\section{Acknowledgement}

Thanks to Dr. Peter Dennis Bolivar Rosa for his support and help in editing this paper.

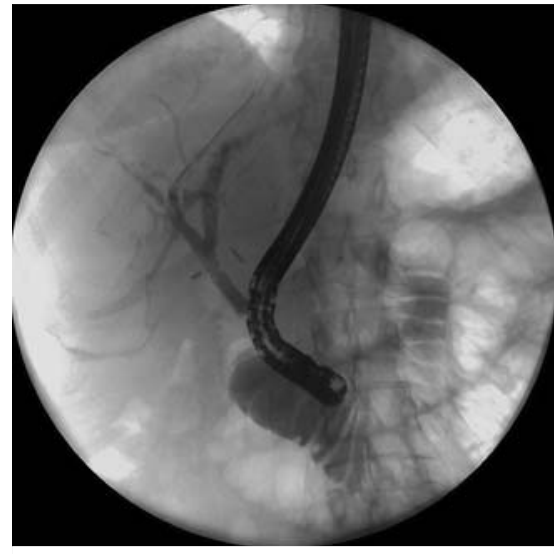

- Fig. 5 Complete resolution of the CBD stricture was observed at cholangiography performed 6 months later.

\section{Competing interests}

Dr. Roberto Di Mitri is consultant to Boston Scientific. The other authors have no conflicts of interest and disclosed no financial relationships relevant to this publication.

\section{The authors}

Roberto Di Mitri ${ }^{1}$, Daniela Scimeca ${ }^{1}$, Michele Amata ${ }^{1}$, Filippo Mocciaro ${ }^{1}$ ๑ Ambra Bonaccorso ${ }^{1}$, Elisabetta Conte ${ }^{1}$, Mario Vallone $^{2}$

1 Gastroenterology and Endoscopy Unit, ARNAS Civico - Di Cristina - Benfratelli Hospital, Palermo, Italy

2 Interventional Radiology Unit, ARNAS Civico - Di Cristina - Benfratelli Hospital, Palermo, Italy

\section{Corresponding author}

\section{Daniela Scimeca, MD, PhD}

Gastroenterology and Endoscopy Unit, ARNAS Civico - Di Cristina - Benfratelli Hospital, Piazza N. Leotta n. 4, 90100 Palermo, Italy

d.scimeca80@gmail.com

\section{References}

[1] Costamagna G, Pandolfi M, Mutignani M et al. Long-term results of endoscopic management of postoperative bile duct strictures with increasing numbers of stents. Gastrointest Endosc 2001; 54: 162-168

[2] Dumonceau JM, Tringali A, Papanikolaou IS et al. Endoscopic biliary stenting: indications, choice of stents, and results: European Society of Gastrointestinal Endoscopy (ESGE) Clinical Guideline - updated October 2017. Endoscopy 2018; 50: 910-930

[3] Hu B, Sun B, Cai Q et al. Asia-Pacific consensus guidelines for endoscopic management of benign biliary strictures. Gastrointest Endosc 2017; 86: 44-58

[4] Nicosia L, Cannataci C, Cortis K et al. Can a multidisciplinary approach improve the care of patients with benign biliary strictures? Gastrointest Endosc 2018; 87: 322-323

\section{Bibliography}

Endoscopy 2022; 54: E608-E609

DOI 10.1055/a-1711-3652

ISSN 0013-726X

published online 26.1.2022

(C) 2022. Thieme. All rights reserved.

Georg Thieme Verlag KG, Rüdigerstraße 14, 70469 Stuttgart, Germany

\section{ENDOSCOPY E-VIDEOS}

https://eref.thieme.de/e-videos

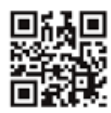

Endoscopy E-Videos is an open access online section, reporting on interesting cases and new techniques in gastroenterological endoscopy. All papers include a high quality video and all contributions are freely accessible online. Processing charges apply (currently EUR 375), discounts and wavers acc. to HINARI are available.

This section has its own submission website at

https://mc.manuscriptcentral.com/e-videos 